\title{
Defervescent dengue patients might be a potential source of infection for vector mosquitoes
}

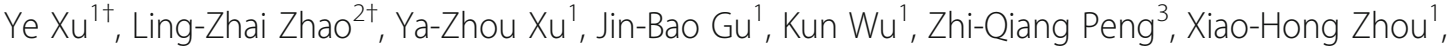 \\ Fu-Chun Zhang ${ }^{2 *}$ and Xiao-Guang Chen ${ }^{1 *}$ (i)
}

\begin{abstract}
Background: Dengue is a re-emerging public health problem and mosquito-borne infectious disease that is transmitted mainly by Aedes aegypti and Ae. albopictus. Early diagnosis, isolation, and treatment of patients are critical steps for dengue epidemic control, especially to prevent secondary transmission of dengue virus (DENV). However, little is known about defervescent dengue patients as a source of infection.

Methods: This case study describes 1268 dengue patients hospitalized at Guangzhou Eighth People's Hospital from June 2013 to December 2014. The viral loads of each individual were measured using real-time reverse

transcription-polymerase chain reaction. Ae. aegypti and Ae. albopictus were exposed to blood meal with gradated dengue viral loads to characterize the relationship between viremia in dengue patients and the vector competence of vector mosquitoes.

Results: The viral numbers in the blood were measured, ranging from $10^{8}$ to $10^{3}$ copies $/ \mathrm{ml}$ from day 1 to day 12 after fever onset. Vector competence analysis of Ae. aegypti and Ae. albopictus indicated that viremia $>10^{4} \mathrm{copies} / \mathrm{ml}$ can still infect vector mosquitoes, which implied that the defervescent dengue patients might be a source of infection.

Conclusions: The results of this study indicate that some defervescent dengue patients still have sufficient viral load to infect vector mosquitoes. Therefore, the protection against mosquito biting for these people should be extended to prevent secondary transmission events.
\end{abstract}

Keywords: Dengue fever, Dengue virus, Vector, Aedes aegypti, Aedes albopictus

\section{Background}

Dengue is a mosquito-borne infectious disease and is transmitted mainly by Aedes aegypti and Ae. albopictus. Early diagnosis, isolation, and treatment of patients are critical steps for dengue epidemic control, especially to prevent secondary transmission of dengue virus (DENV) [1, 2]. The host and virus variables are associated with DENV transmission from symptomatic dengue cases to vector mosquitoes [3].

\footnotetext{
*Correspondence: xgchen2001@hotmail.com; gz8hzfc@126.com

${ }^{\dagger}$ Ye Xu and Ling-Zhai Zhao contributed equally to this work.

${ }^{2}$ Guangzhou Eighth People's Hospital, Guangzhou Medical University, Guangzhou, China

'Department of Pathogen Biology, School of Public Health, Southern Medical University, Guangzhou, China

Full list of author information is available at the end of the article
}

Moreover, at a given level of viremia, DENV-infected people with no detectable symptoms or not yet exhibiting symptoms are significantly more infectious to mosquitoes than people with symptomatic infections [4]. At present, the discharge criteria for dengue patients set by the WHO and China are as follows: no fever for 24-48 h, improvement in clinical status, increasing trend of platelet count, and stable hematocrit without intravenous fluids $[5,6]$. Is it possible for defervescent dengue patients to be a source of infection? What is the changing role of viremias in dengue patients after fever onset? Is there any minimum DENV copy number required to infect vector mosquitoes? To answer these questions, we analyzed the viremias in 1268 dengue patients ranging from

(C) The Author(s). 2020 Open Access This article is distributed under the terms of the Creative Commons Attribution 4.0 International License (http://creativecommons.org/licenses/by/4.0/), which permits unrestricted use, distribution, and 
day 1 to day 12 after fever onset and tested the infection rates of various viral loads of DENV in blood meals on vector mosquitoes.

\section{Methods}

\section{Study design}

Dengue patients hospitalized at Guangzhou Eighth People's Hospital from June 2013 to December 2014 were included in this study. The diagnosis of dengue patients was performed in accordance with the 2009 WHO guidelines for dengue diagnosis, treatment, prevention, and control. Inclusion criteria were patients positive for DENV RNA, as determined by reverse transcriptionpolymerase chain reaction (RT-PCR), and nonstructural protein 1 (NS1) or virus-specific IgM antibody, as determined by enzyme-linked immunosorbent assay (ELISA) test.

\section{Sample size determination}

Blood samples of 3-5 $\mathrm{ml}$ were collected, and serum was separated by centrifugation. In total, 1268 cases of dengue patients hospitalized at Guangzhou Eighth People's Hospital from June 2013 to December 2014 were included in this study. All infections belonged to serotype I. The median age was 48 years (range $17-90$ years). The male/female ratio was $1: 0.83$ (693 male cases and 575 female cases). A total of 1152 cases were classified as dengue fever, and 116 were severe dengue. Overall, 1955 serum samples were collected for viral load detection (some patients provided several serum samples at different time points).

\section{Viral load quantification}

DENV loads were determined using the DENV1-4 OneStep Real-Time RT-PCR Kit (DaAn Gene, Guangzhou, China) following the manufacturer's instructions. Each sample was tested at least in duplicate, and each experiment was performed twice and included negative and positive controls. The limit of detection was 100 copies $/ \mathrm{ml}$.

\section{Ae. aegypti and Ae. albopictus treated with blood meals with gradated dengue viral loads}

We used artificial blood meals with graded dengue viral loads to explore the relationship between viremia in dengue patients and the infection of vector mosquitoes, Ae. aegypti and Ae. albopictus, according to the methods described in previous studies [7-9]. In brief, infectious blood meals were prepared by mixing defibrinated sheep blood (Solarbio, Beijing, China) with gradated fresh DENV viral suspension at a ratio of $1: 2$. For treatment, blood meals with a tenfold dilution series of viral load $\left(10^{10}\right.$ to $10^{4}$ copies $\left./ \mathrm{ml}\right)$ were used. Each blood meal was warmed to $37^{\circ} \mathrm{C}$ and transferred into a Hemotek blood feeding system (Discovery Workshops, Blackburn, UK) for mosquito feeding. Five days after eclosion, $A e$. aegypti and Ae. albopictus females were used. After exposure for $30 \mathrm{~min}$, a total of 2486 Ae. albopictus and $664 \mathrm{Ae}$. aegypti were anesthetized with ether, and fully engorged females were selected for further testing. The infection rates of Ae. albopictus on the 4th day post infection (dpi) $(n=1222)$ and the 14th dpi $(n=1264)$ and Ae. aegypti on the 4th dpi $(n=371)$ and 14th dpi $(n=$ 293) after blood feeding were determined by PCR with DENV-specific primers. Experiments were performed in accordance with standard procedures in a Biosafety Level 2 laboratory.

\section{Results}

\section{Viral load throughout illness course}

The kinetics of the percentage of patients with fever throughout the illness course, viral load of different patients throughout the illness course, and percentage of patients with positivity for DENV RNA are summarized in Fig. 1. All patients had fever at the onset of disease, which lasted 5-6 days in most cases. The viral load gradually decreased as patients recovered from the disease. The percentage of patients who were positive for DENV RNA also declined from $100 \%$ on day 1 to $23.53 \%$ on day 12 after disease onset. Dengue viremia kinetics in dengue patients suggested that the maximum observed viral load reached an average of $10^{8}$ copies $/ \mathrm{ml}$ on the first day of fever. Then, the viral load gradually decreased over the days with fever. The viral load decreased to an average of $10^{3}$ copies $/ \mathrm{ml}$ on day 12 after fever.

\section{Vector competence under gradated viral loads of $A e$. aegypti and Ae. albopictus}

The infection rates in both Ae. aegypti and Ae. albopictus decreased as the viral load decreased in the blood meal. For Ae aegypti, the infection rates reached $100 \%$ when the viral load in the blood meal was $10^{10}$ copies/ $\mathrm{ml}$ and $10^{9}$ copies $/ \mathrm{ml}$ on the 14 th dpi. When the viral loads were below $10^{6}$ copies $/ \mathrm{ml}$, the infection rates became $0 \%$ on the 4 th dpi. However, the viral load of $10^{4}$ copies $/ \mathrm{ml}$ could still infect Ae. aegypti with an average infection rate of $9.4 \%$ on the 14th dpi after the blood meal. For Ae albopictus, when the viral load of a blood meal was $10^{10}$ copies $/ \mathrm{ml}$, both the infection rates on the 4th dpi and 14th dpi were more than 95\%. However, when the viral loads were below $10^{5}$ copies $/ \mathrm{ml}$, the infection rates became $0 \%$ on either the 4 th dpi or the 14th dpi after the blood meal. These results indicated that it is possible to infect Ae. aegypti and Ae. albopictus when the viral copy number in viremia was greater than $10^{4}$ copies $/ \mathrm{ml}$ or $10^{6}$ copies $/ \mathrm{ml}$, respectively. 


\section{A}

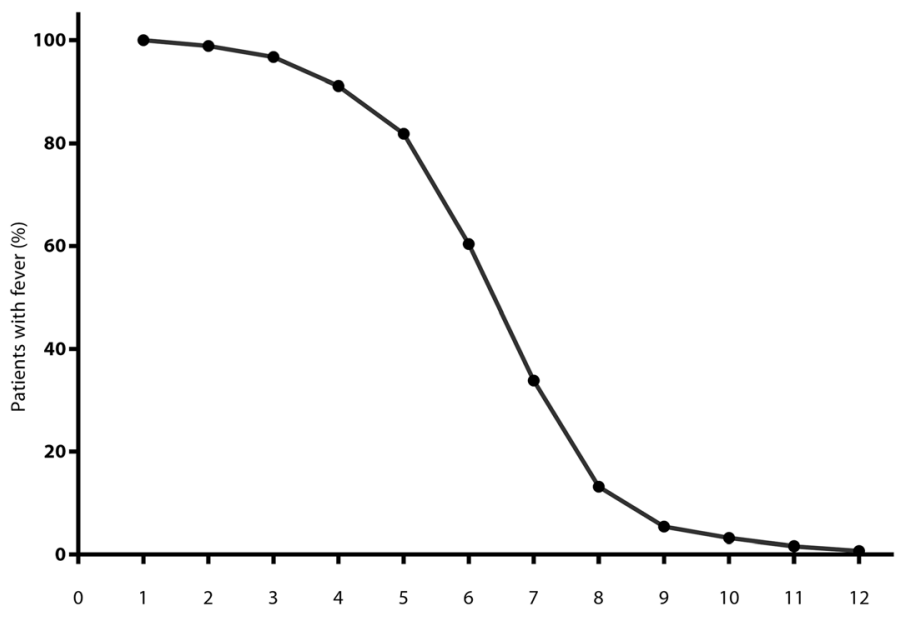

B

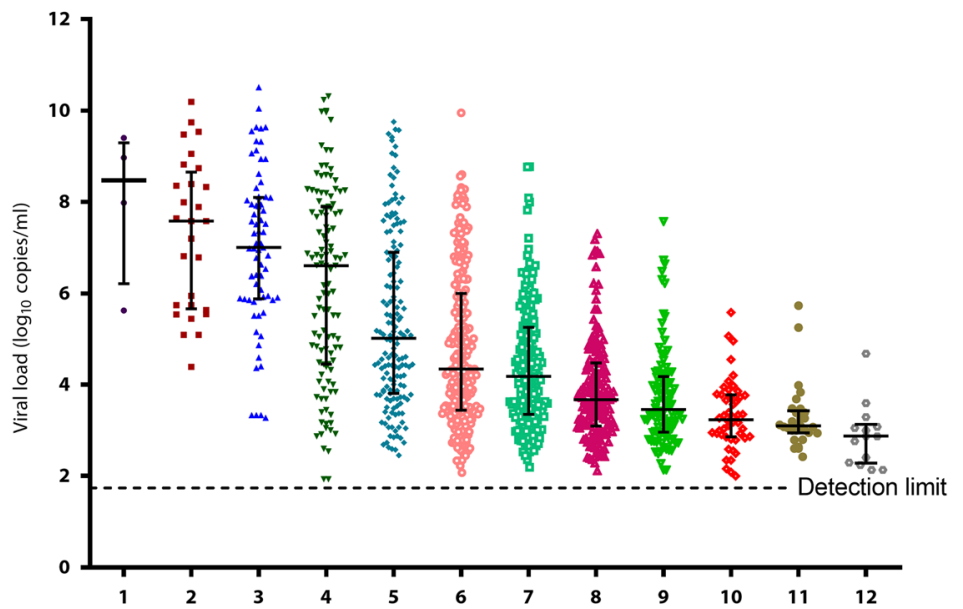

c

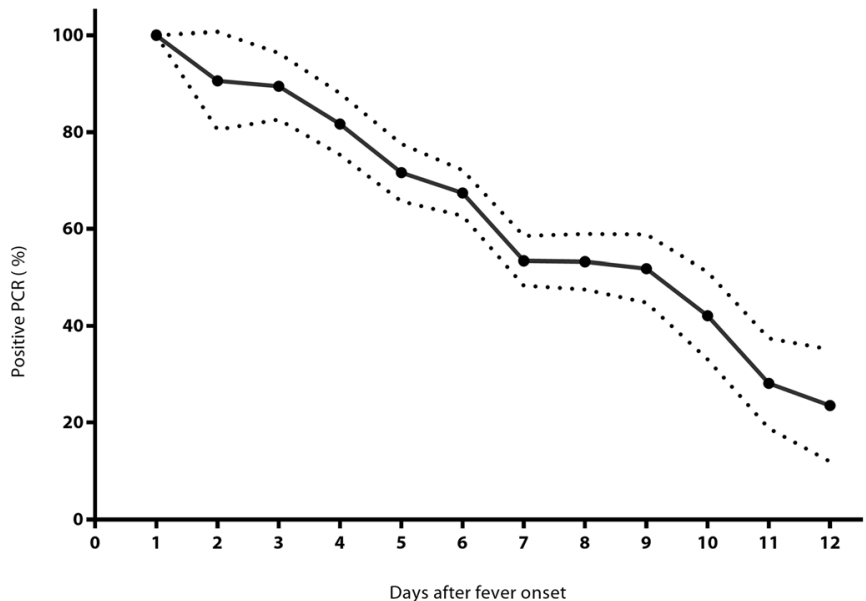

Fig. 1 Characterization of 1268 dengue patients after fever onset. a Percentage with fever. $\mathbf{b}$ Viral load. Each dot represents the virus copies of individual patients. Error bars indicate SDs. c Percentage of positive PCR. reverse transcription-polymerase chain reaction (RT-PCR) 
A

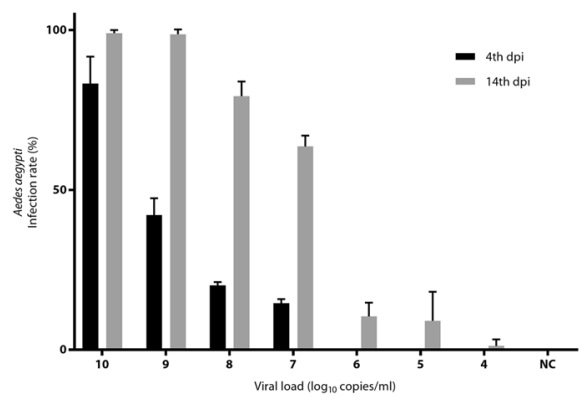

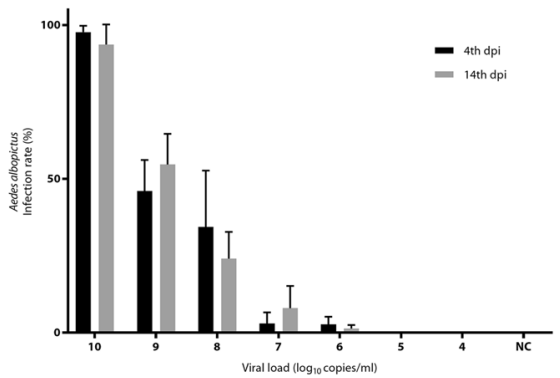

Fig. 2 Infection rates of a: Ae. aegypti and $\mathbf{b}$ : Ae. albopictus fed on blood with various dengue viral copy numbers. The infection rates were measured 4th (black) and 14th dpi (gray) after the blood meal. Error bars indicate SDs. NC stands for negative control without virus in the blood meal. All experiments were repeated three times. Dpi: Day post infection

\section{Discussion}

In this study, the infection rates on the 4th dpi and 14th dpi of both Ae. aegypti and Ae. albopictus were measured. On the 4th dpi, DENV began to spread to other organizations, which indicated an early infectious status after infection. On the 14th dpi, DENV was detected in the midgut, salivary grand, ovary, and head, which indicated a stable infectious status after infection. To set a rigid gradient virus titer and simulate the infection events, we used artificial blood feeding. Although artificial blood feeding could not present the same condition as natural human blood, it could obtain controllable treatments and reliable results and has been used in many studies for infection assays [7].

Most of the 1268 dengue patients exhibited fever for 5-6 days and were discharged 1-2 days after defervescence. Our results showed that viral loads in 1268 dengue patients gradually decreased from $10^{8}$ to $10^{3}$ copies $/ \mathrm{ml}$ after fever onset (Fig. 1), and vector mosquitoes, Ae. aegypti and Ae. albopictus, can still be infected when the viral copy number is greater than $10^{4}$ copies/ $\mathrm{ml}$ and $10^{6}$ copies $/ \mathrm{ml}$ in the blood meal, respectively (Fig. 2). However, the viral load of $70.4 \%$ (112/159) of patients on day 5 after fever onset was above $10^{4}$ copies $/ \mathrm{ml}$, which means that the viremia in these patients could be an infectious source of dengue virus. On day 8 after fever onset, approximately $85 \%$ of patients did not have fever, but the viral load of $35.8 \%$ (58/162) of patients was above $10^{4}$ copies $/ \mathrm{ml}$, which is sufficient to infect vector mosquitoes. On day 12 after fever onset, there were no patients with fever, and only $7 \%(1 / 14)$ had a viral load above $10^{4}$ copies $/ \mathrm{ml}$. According to our results, a virus load below $10^{6}$ copies $/ \mathrm{ml}$ could not infect Ae. aegypti on the 4th dpi and below $10^{4}$ copies $/ \mathrm{ml}$ on the 14th dpi. A virus load below $10^{5}$ copies $/ \mathrm{ml}$ could not infect Ae. albopictus on the 4th dpi and the 14th dpi. In the field, Ae. aegypti and Ae. albopictus can survive for more than one month. The viral load of a few patients was still above $10^{4}$ copies/ml on day 12 after fever onset. Currently, the discharge criteria for dengue patients, as set by the WHO and China, are as follows: no fever for $24-48 \mathrm{~h}$, improvement in clinical status, increasing trend of platelet count, and stable hematocrit without intravenous fluids $[5,6]$. These criteria do not guarantee that viral loads are insufficient to infect vector mosquitoes. This possibility would provide an opportunity to infect vectors and enable a secondary epidemic.

Our results are highly reliable and consistent with previous studies describing the various viral loads in dengue patients at different clinical stages and demonstrating that a certain number of DENV copies are required for the infection of vector mosquitoes $[3,4,10]$. These results implied that the viremia in patients after defervescence might be a source of infection, but this possibility becomes increasingly faint as more time passes without fever.

\section{Conclusions}

The results of this study indicate that some defervescent dengue patients still have sufficient viral load to infect vector mosquitoes. Therefore, the protection against mosquito biting for these patients should be extended, by seven days after defervescence, for example, to reduce the potential infectious sources of dengue.

\section{Abbreviations \\ DENV: Dengue virus: ELISA: Enzyme-linked immunosorbent assay; \\ NS1: Nonstructural protein 1; RT-PCR: Reverse transcription-polymerase chain reaction; WHO: World Health Organization}

Acknowledgements

Not applicable.

Authors' contributions

$Y X$ and LZZ contributed equally to this article. XGC and FCZ designed the study. YX, LZZ, YZX, JBG, ZQP and XHZ contributed to data collection. All authors analyzed and interpreted the data. All authors read and approved the final version of the manuscript. 


\section{Author's information}

$Y X$ is a Postdoctoral Fellow from the Department of Pathogen Biology, School of Public Health of Southern Medical University, Guangzhou, China. LZZ is from Guangzhou Eighth People's Hospital, Guangzhou Medical University, Guangzhou, China. YZX is the Master Student from the Department of Pathogen Biology, School of Public Health of Southern Medical University, Guangzhou, China. JBG, KW, XGC are Professors from the Department of Pathogen Biology, School of Public Health of Southern Medical University, Guangzhou, China. ZQP is a Professor from Guangdong Provincial Center of Disease Control and Prevention, Guangzhou, China. XHZ is a Professor from Guangzhou Eighth People's Hospital, Guangzhou Medical University, Guangzhou, China.

\section{Funding}

This research was supported by a combination of funding from the National Natural Science Foundation of China (81420108024, 31830087), the National Institutes of Health, USA (Al136850), the Natural Science Foundation of Guangdong Province (2014A030312016), and the Guangzhou Synergy Innovation Key Program for Health (201803040006).

\section{Availability of data and materials}

No data and material are available for sharing.

\section{Ethics approval and consent to participate}

The ethics committee of Guangzhou Eighth People's Hospital, Guangzhou, China, approved this research, and all patients provided written informed consent.

\section{Consent for publication}

Not applicable.

\section{Competing interests}

The authors declare that they have no competing interests.

\section{Author details}

'Department of Pathogen Biology, School of Public Health, Southern Medical University, Guangzhou, China. ${ }^{2}$ Guangzhou Eighth People's Hospital, Guangzhou Medical University, Guangzhou, China. ${ }^{3}$ Guangdong Provincial Center of Disease Control and Prevention, Guangzhou, China.

Received: 11 August 2019 Accepted: 20 January 2020

Published online: 02 March 2020

\section{References}

1. Bhatt S, Gething PW, Brady OJ, Messina JP, Farlow AW, Moyes $C L$, et al. The global distribution and burden of dengue. Nature. 2013;496(7446):504.

2. Brady OJ, Gething PW, Bhatt S, Messina JP, Brownstein JS, Hoen AG, et al. Refining the global spatial limits of dengue virus transmission by evidencebased consensus. PLoS Negl Trop Dis. 2012;6(8):e1760.

3. Nguyen NM, Kien DTH, Tuan TV, Quyen NTH, Tran CN, Thi LV, et al. Host and viral features of human dengue cases shape the population of infected and infectious Aedes aegypti mosquitoes. Proc Natl Acad Sci U S A. 2013;110(22):9072-7.

4. Duong V, Lambrechts L, Paul RE, Ly S, Lay RS, Long KC, et al. Asymptomatic humans transmit dengue virus to mosquitoes. Proc Natl Acad Sci U S A. 2015;112(47):14688-93

5. World Health Organization. Denque: guidelines for diagnosis, treatment, prevention and control: World Health Organization; 2009. http://whqlibdoc. who.int/publications/2009/9789241547871_eng.pdf. Accessed 12 July 2019.

6. National Health Commission of the People's Republic of China. Guidelines for diagnosis and treatment of dengue. 2014. http://www.nhc.gov.cn/yzygj/ s3593g/201410/d417aa2e783949e48f8a7366d7fdfacc.shtml. Accessed 12 July 2019.

7. Liu Z, Zhou T, Lai Z, Zhang Z, Jia Z, Zhou G, et al. Competence of Aedes aegypti, Ae. albopictus, and Culex quinquefasciatus mosquitoes as Zika virus vectors, China. Emerg Infect Dis. 2017;23(7):1085.

8. Ledermann JP, Guillaumot L, Yug L, Saweyog SC, Tided M, Machieng P, et al. Aedes hensilli as a potential vector of Chikungunya and Zika viruses. PLoS Negl Trop Dis. 2014;8(10):e3188.
9. Liu Z, Zhang Z, Lai Z, Zhou T, Jia Z, Gu J, et al. Temperature increase enhances Aedes albopictus competence to transmit dengue virus. Front Microbiol. 2017;8:2337.

10. Whitehorn J, Kien DTH, Nguyen NM, Nguyen HL, Kyrylos PP, Carrington LB, et al. Comparative susceptibility of Aedes albopictus and Aedes aegypti to dengue virus infection after feeding on blood of viremic humans: implications for public health. J Infect Dis. 2015;212(8):1182-90.

\section{Ready to submit your research? Choose BMC and benefit from:}

- fast, convenient online submission

- thorough peer review by experienced researchers in your field

- rapid publication on acceptance

- support for research data, including large and complex data types

- gold Open Access which fosters wider collaboration and increased citations

- maximum visibility for your research: over $100 \mathrm{M}$ website views per year

At BMC, research is always in progress.

Learn more biomedcentral.com/submissions 